\title{
Prediction of Ergogenic Mouthguard Effects in Volleyball: A Pilot Trial
}

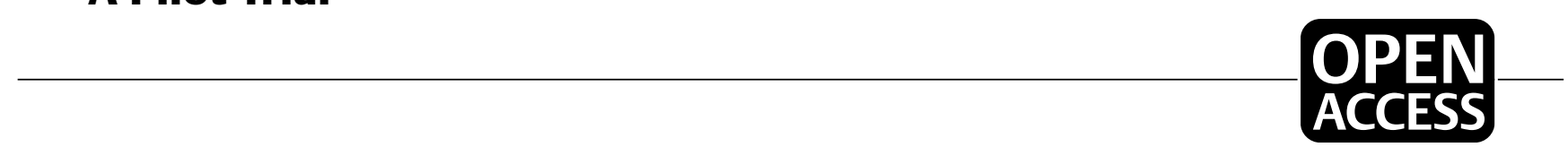

(ㄷ) (i) $(9)$

\author{
Authors \\ Antina Schulze, Martin Busse
}

\begin{abstract}
Affiliations Leipzig, Leipzig, Germany

Keywords tion, EMG

$\begin{array}{ll}\text { received } & 28.06 .2019 \\ \text { revised } & 10.10 .2019 \\ \text { accepted } & 14.10 .2019\end{array}$
\end{abstract}

Institute of Sports Medicine \& Prevention, University of

volleyball, mouthguard, pinpoint accuracy, body stabiliza-

Bibliography

DOI https://doi.org/10.1055/a-1036-5888

Sports Medicine International Open 2019; 3: E96-E101

(c) Georg Thieme Verlag KG Stuttgart · New York

ISSN 2367-1890

\section{Correspondence}

Dr. Antina Schulze

Institute of Sports Medicine

University of Leipzig

Marschnerstraße 29

04109 Leipzig

Germany

Tel : +493419731741, Fax : +493419731748

a.schulze@uni-leipzig.de

\begin{abstract}
Dental occlusion may affect static and dynamic balance. The effects of a mouthguard on pinpoint accuracy in volleyball were investigated in 28 players who completed a volleyball specific test. Also, masticatory electromyographic tests were performed. The mean pinpoint accuracy was significantly higher with a mouthguard $(68.6 \pm 9.3$ vs. $64.0 \pm 7.0$ points from 100 ; $p<0.006)$. However, differential mouthguard effects were seen, and three subgroups were classified: Group 1 (markedly improved pinpoint accuracy), Group 2 (improved pinpoint accuracy), and Group 3 (reduced pinpoint accuracy). Group 1 had a high masseter resting tone, the masseter activity was low in MVC (maximum voluntary clench) and increased in BOC (maximum bite on cotton rolls; $p<0.04$ ). This indicates a masseter weakness, which would be compensated by a mouthguard. In Group 2, the masseter activity in MVC was high-normal with an imbalance which was improved in BOC $(p<0.01)$, indicating a possible mouthguard benefit. In Group 3, MVC and BOC were in a high-normal range and showed no relevant deficits. In these subjects the mouthguard had adverse effects. Overall, subjects with masticatory deficits had a benefit from the mouthguard in pinpoint accuracy. Positive or negative mouthguard responders may be detectible from electromyographic tests.
\end{abstract}

\section{Introduction}

It is suggested that a relationship between the masticatory system and the body movement system exists [1]. Studies showed that different jaw positions caused changes in gait stability while running with increasing speeds [2,3]. A functional correlation was found between the trigeminal and cervical system, with reciprocal co-activations and inhibitions of the jaw, neck, shoulder, and limb muscles during performance [4-8]. The mandibular proprioception, assisted by the trigeminal nerve and mediated by the masticatory muscles and periodontal ligament, contributes to head posture control via the sternocleidomastoid muscles [9]. Several studies showed that teeth clenching may play an important role in rapid body posture stabilization [10-12], gait stabilization [2], posture stability, and balance control [13]. The stomatognathic system may influence the muscle functions in other parts of the body, e. g., the range of hip movement [5], the visual stabilization quality [14], and the head posture [9]. Julià-Sánchez et al. [15] compared the body balance on unstable platforms under opposing dental occlusion and fatigue conditions for two extreme levels of stability (stable/ unstable). They concluded that the sensory information for balance control is linked to the dental occlusion and comes strongly into effect in more difficult conditions such as instability and fatigue.

It is speculated whether disharmonies in dental occlusion can modify muscle functions throughout the body. Occlusal interferences, either natural or experimental, may impair the function of the jaw muscles and temporomandibular joints [7, 16, 17]. The loss 
of occlusal support and the instability of the mandibular position might influence weight distribution and may cause reduced agility [18].

Based on these findings, it is the study hypothesis that mouthguard use may have positive effects on the intermuscular coordination in sports for two reasons:

1. Mouthguards may facilitate powerful jaw-clenching and a subsequent concurrent activation potentiation through the remote voluntary contraction of the mandible muscles.

2. Mouthguards may compensate for muscle imbalances or pathologies in the temporomandibular system.

Volleyball requires specific abilities and skills. The motor output is composed of the conditional abilities, as well as the coordinative capabilities of coupling, orientation, balance, adaptation, rhythmization and differentiation. The coordinative ability includes the precision of movement and thus the pinpoint accuracy of the athlete. Owing to these complexities, the pinpoint accuracy in volleyball was used in the current study as a model for mouthguard effects on intermuscular coordination. To the best of our knowledge, no previous study has analyzed mouthguard effects in a sport-specific volleyball test.

\section{Materials and Methods}

\section{Subjects}

A total of 28 healthy subjects, 15 women and 13 men, 19 to 39 years old (mean age: $25.2 \pm 5.2$ years; height: $177.9 \pm 8.3 \mathrm{~cm}$; weight: $73.0 \pm 9.1 \mathrm{~kg}$ ) participated in this study. The subjects completed an inclusion criteria questionnaire. Only right-handed individuals with a complete and sound permanent dentition; no periodontal problems; no prostheses or reconstructions; and no orthopedic, myogenic, or arthrogenic complaints related to temporomandibular dysfunctions were considered for participation. Subjects with past orthopedic and present acute or chronic complaints were excluded.

The study was approved by the Institutional Review Board and Human Research Committee, No. 445-15-21122015. The subjects were informed of the benefits and risks of the investigation prior to signing the institutionally approved informed consent document to participate in the study. The study conformed to the Standards for Ethics in Sport and Exercise Science Research [19] and required players to provide informed consent before participation. None of the subjects reported former use of a mouthguard.

\section{Mouthguard}

A "vented boil-and-bite" mouthguard was used (Nike, Beaverton, OR, USA). This mouthguard (MG) consists of a special flexible, shock-absorbent plastic. The MG was placed in boiling water for 30 $s$ and then carefully placed in the subject's mouth to cover the upper teeth. The subject was instructed to bite down. Moderate pressure was placed on the lips and cheeks for $30 \mathrm{~s}$. The MG was then removed and rinsed in cold water.

\section{SinfoMed K7-system}

A myofunctional examination was carried out with the SinfoMed K7-system (SinfoMed, Frechen, Germany). It measures masticatory muscle tension and neuromuscular activities. Using bipolar sur- face electrodes, electromyographic (EMG) data can be taken from several muscle sites simultaneously. The EMG activities of the following muscle groups were recorded: temporalis, masseter, cervical group, and digastrics.

The EMG activity was recorded in three different tests:

1. Resting tone of the four muscle groups at both sides for $14 \mathrm{~s}$. This test was repeated three times and the data of the third test were used.

2. Maximum voluntary clench (MVC) in the intercuspal position: the subjects had to clench two times as hard as possible and maintain the level of contraction for $2 \mathrm{~s}$ with an intervening $1 \mathrm{~s}$ break between clenches.

3. Maximum bite on cotton rolls (BOC) positioned on the lateral teeth: the subjects had to clench two times as hard as possible and maintain the level of contraction for $2 \mathrm{~s}$ with an intervening $1 \mathrm{~s}$ break between clenches.

For all tests, the subjects were placed on a chair and had to maintain a natural, erect and relaxed position without a head or neck support.

\section{Interpretation of the masticatory EMG tests}

Experiment 1 (resting tone) assessed muscular output such as fatigue, hyperactivity, and passivity. A tone above threshold (masseter $>2 \mu \mathrm{V}$; temporalis $>2.2 \mu \mathrm{V}$; digastrics $>1.7 \mu \mathrm{V}$ ) indicates a muscle overload or muscle weakness, respectively.

In experiments 2 and 3, the MVC normal range is $220-250 \mu \mathrm{V}$. MVC and BOC are function tests and interpreted as follows: a) MVC below threshold indicates intercuspal disorder and muscular weakness; b) side differences indicate muscular imbalance in MVC or BOC.

In experiment 3, higher BOC values indicate an improvement of muscular imbalance, e.g., due to a compensation of intercuspal disturbance by the cotton rolls. Lower BOC values indicate muscle fatigue.

\section{Volleyball testing}

The sport-specific testing included ten offensive and defensive volleyball components, such as passing (short, medium, long), digging (right, center, left), and servicing (back right, back center, back left, short). The participants performed these exercises ( $\mathbf{F i g . ~ 1 )}$ with and without an MG in a randomized order. Different marked target fields had to be hit ( $\triangleright$ Fig. 1). The number of hits was recorded (maximum 100 points per each test with and without mouthguard). Finally, the sum of hits was calculated separately for mouthguard (MG) and no mouthguard (NoMG) use. The test sessions were performed on one day in an indoor team sports hall. The net height was $2.24 \mathrm{~m}$ for women and $2.43 \mathrm{~m}$ for men.

\section{Statistical analysis}

All data are presented as the means $\pm S D$. A paired t-test was performed to compare the results with the MG vs. no MG use. The Mann-Whitney U-test was used for the comparison of group differences. A p-value < 0.05 was considered significant, and a p-value < 0.005 was considered highly significant. Statistical analyses were performed using GraphPadInStat software (GraphPad Software, La Jolla, CA, USA). 


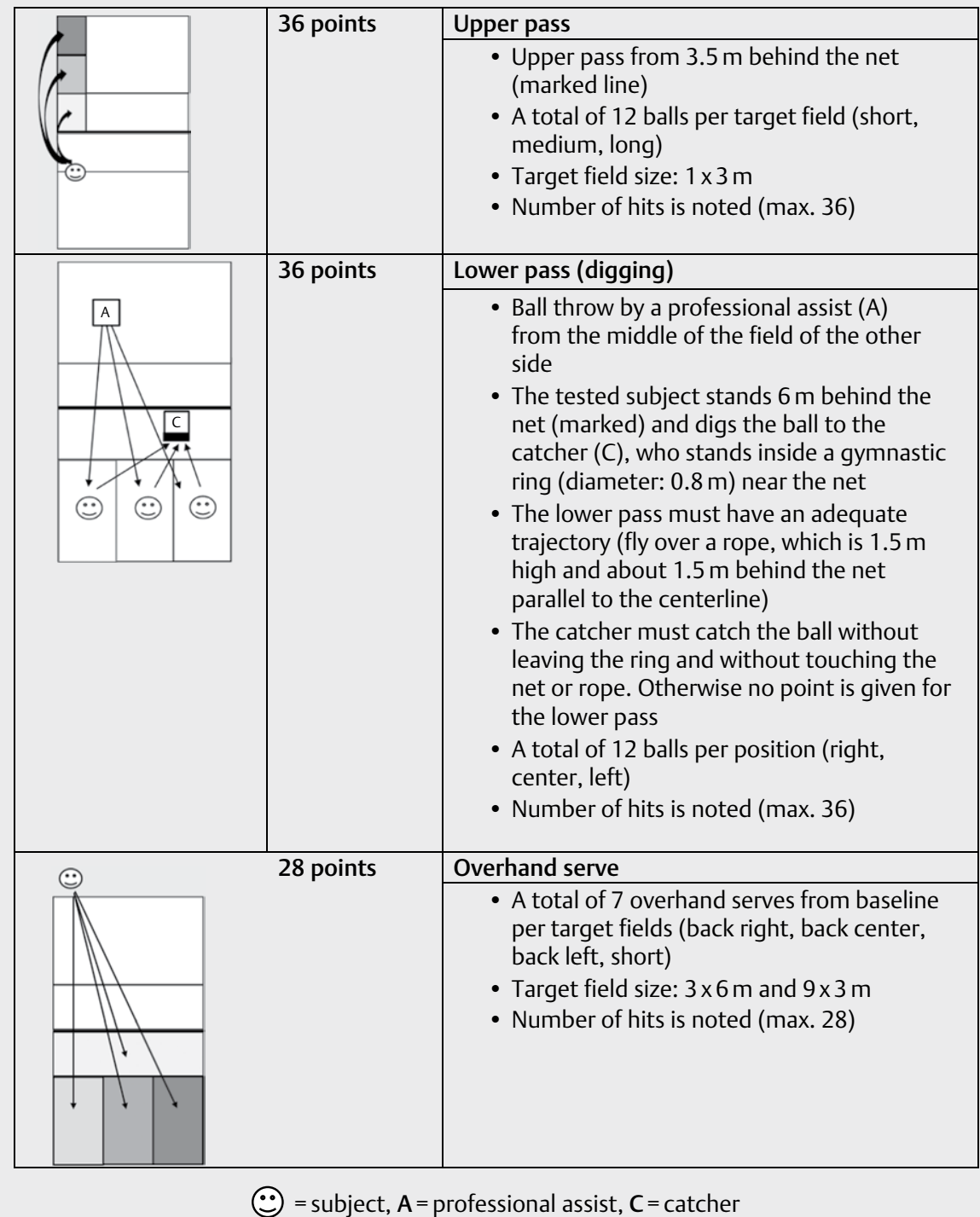

Fig. 1 Volleyball test protocol

\section{Results}

\section{Pinpoint accuracy}

The pinpoint accuracy (PA) of the total group $(n=28)$ was significantly higher when using an MG vs. NoMG (68.6 \pm 9.3 vs. $64.0 \pm 7.0$ points from 100; $\mathrm{p}<0.006)$. However, some subjects performed worse, some better, and some markedly better with an MG. Accordingly, three subgroups were distinguished. The criterion was a pinpoint count difference between the individual results with an MG and NoMG of at least 5 points ( $\geq 50 \%$ of the standard deviation). A difference of less than 5 points was therefore omitted ( 5 subjects). Four participants did not come for the SinfoMed K7measurement. The point count of Group 1 was two digits better, with an $M G(n=5 ; 76.1 \pm 10.0$ vs. $61.0 \pm 9.0$ points; $p<0.016)$. The point count of Group 2 was one digit better, with an MG ( $n=9$; $70.1 \pm 7.6$ vs. $64.4 \pm 7.8$ points; $p<0.001$ ). The point count of Group
3 was reduced, with an MG ( $n=6 ; 59.7 \pm 5.5$ vs. $66.0 \pm 4.2$ points; $\mathrm{p}<0.0008)$.

\section{Masticatory resting EMG}

The results of the masticatory resting EMG of the three subgroups are shown in $>$ Table 1 . The masseter resting tone was increased in Group 1 (threshold $2 \mu \mathrm{V}$ ). The resting tone of the digastric muscles was increased in all groups (threshold $1.7 \mu \mathrm{V}$ ). No increased resting tone of the cervical or temporalis groups was found in any group (threshold $2.2 \mu \mathrm{V}$ ) ( Table 1). When comparing the muscle tones of each side, only the left masseter tone of Group 1 was significantly higher than in Group 2 ( $p<0.03)$. In a comparison of the cumulative values of all masticatory muscles, Group 2 had a significantly lower resting tone vs. Group 1 ( $p<0.022)$ and vs. Group $3(p<0.015)$.

Maximum voluntary clenching (MVC) and bite on cotton rolls $(\mathrm{BOC})$ 
> Table 1 The electromyographic (EMG) resting tones of the muscle groups temporalis anterior, masseter, cervical group, and digastrics.

\begin{tabular}{|c|c|c|c|}
\hline & Group $1 \mathrm{n}=5$ & Group $2 \mathrm{n}=9$ & Group $3 n=6$ \\
\hline & \multicolumn{3}{|c|}{$\mu \mathrm{V}$} \\
\hline Left temporalis & $2.04(0.27)$ & $2.50(1.30)$ & $2.70(0.75)$ \\
\hline Right temporalis & $1.78(0.61)$ & $1.73(0.73)$ & $2.32(1.11)$ \\
\hline Mean value temporalis & $1.91(0.38)$ & $2.11(0.89)$ & $2.51(0.56)$ \\
\hline Mean side difference temporalis & $0.34(0.50)$ & $1.03(0.87)$ & $1.25(0.79)$ \\
\hline Left masseter & $2.42(1.02)^{*}$ & $1.26(0.44)^{*}$ & $1.98(0.97)$ \\
\hline Right masseter & $2.44(0.93)$ & $1.51(0.68)$ & $1.95(0.62)$ \\
\hline Mean value masseter & $2.43(0.86)$ & $1.38(0.55)$ & $1.97(0.78)$ \\
\hline Mean side difference masseter & $0.51(0.58)$ & $0.30(0.27)$ & $0.40(0.22)$ \\
\hline Left cervicalis & $2.90(1.32)$ & $1.74(0.83)$ & $1.95(0.80)$ \\
\hline Right cervicalis & $1.82(0.79)$ & $1.75(1.32)$ & $2.02(0.53)$ \\
\hline Mean value cervicalis & $1.70(0.95)$ & $1.75(0.97)$ & $1.98(0.51)$ \\
\hline Mean side difference cervicalis & $1.08(1.06)$ & $0.70(0.74)$ & $0.83(0.79)$ \\
\hline Left digastric & $2.02(0.49)$ & $2.37(1.32)$ & $2.03(0.71)$ \\
\hline Right digastric & $1.82(0.22)$ & $2.23(1.07)$ & $2.37(0.74)$ \\
\hline Mean value digastric & $1.92(0.33)$ & $2.30(1.16)$ & $2.05(0.93)$ \\
\hline Mean side difference digastric & $0.32(0.23)$ & $0.49(0.44)$ & $0.47(0.31)$ \\
\hline Mean activity of all masticatory muscles & $2.16(0.81)$ & $1.89(1.05)^{*}$ & $2.16(0.78)$ \\
\hline
\end{tabular}

> Table 2 Electromyographic activity of the temporalis anterior and masseter muscles; two times in maximum voluntary clench (MVC $1+$ MVC 2) and two times in maximum bite on cotton rolls (BOC $1+$ BOC 2$)$.

\begin{tabular}{|l|l|l|l|}
\hline & Group 1 & Group 2 & Group 3 \\
\cline { 2 - 4 } & & \multicolumn{1}{|c|}{$\mathbf{M V}$} \\
\hline Left temporalis MVC & $173.1(51.38)$ & $181.73(73.75)$ & $253.68(120.99)$ \\
\hline Right temporalis MVC & $155.15(66.30)$ & $240.55(95.93)$ & $243.04(101.58)$ \\
\hline Temporalis MVC & $164.13(56.71)$ & $211.14(88.35)$ & $248.36(106.65)$ \\
\hline Left masseter MVC & $172.51(93.61)$ & $229.10(179.29)$ & $230.08(69.06)$ \\
\hline Right masseter MVC & $168.44(111.40)$ & $255.11(195.43)$ & $252.62(86.86)$ \\
\hline Masseter MVC & $170.48(97.03)$ & $242.10(182.42)$ & $241.35(75.73)$ \\
\hline Mean MVC of temporalis and masseter & $167.30(77.42)$ & $226.62(142.13)$ & $244.85(90.53)$ \\
\hline Left temporalis BOC & $135.30(69.32)$ & $223.69(107.53)$ & $226.26(113.11)$ \\
\hline Right temporalis BOC & $133.96(67.23)$ & $259.12(98-04)$ & $214.52(61.99)$ \\
\hline Temporalis BOC & $134.63(64.38)$ & $241.40(101.47)$ & $220.39(87.18)$ \\
\hline Right masseter BOC & $209.18(126.11)$ & $305.24(164.83)$ & $244.14(73.51)$ \\
\hline Left masseter BOC & $230.94(160.47)$ & $319.29(199.68)$ & $246.92(99.16)$ \\
\hline Masseter BOC & $220.06(136.34)$ & $312.27(177.77)$ & $245.53(83.23)$ \\
\hline Mean BOC of temporalis and masseter & $177.35(112.76)$ & $276.84(147.11)$ & $232.96(84.34)$ \\
\hline SD in brackets. & & & \\
\hline
\end{tabular}

In Group 1, the cumulative masticatory activity (masseter and temporalis) was reduced below the threshold in MVC and BOC. In BOC, the temporalis activity decreased insignificantly and the masseter activity increased significantly $(p<0.04)$ in relation to MVC ( Table 2).

In Group 2, the cumulative masticatory activity in MVC was in the normal threshold range and increased significantly in BOC $(p<$ 0.009 ). The temporalis activity was in the normal MVC range and increased insignificantly in BOC. The masseter activity in MVC was in the normal threshold range and increased significantly in $\mathrm{BOC}$ to $>300 \mu \mathrm{V}(\mathrm{p}<0.012$; $>$ Table 2$)$.

Group 3 showed similar activities in MVC and BOC, both within the threshold range ( $\triangleright$ Table 2 ).

Comparison of the masticatory activity in MVC and BOC within the subgroups

In MVC, the cumulative masticatory activity was significantly lower in Group 1 vs. Group 3 ( $p<0.005)$. In BOC, the cumulative masticatory activity in Group 1 was significantly lower vs. Group 2 $(p<0.013)$ and also vs. Group $3(p<0.035)$ 
Comparison of the temporalis and masseter activity in MVC and BOC within the subgroups

In Group 1, temporalis activity was below the threshold (200$250 \mu \mathrm{V}$ ) in MVC and was even lower (though insignificantly) in BOC ( $\triangleright$ Table 2). Masseter activity was below the threshold in MVC and increased markedly in BOC to values within the threshold $(p<0.04$, - Table 2).

In Group 2, the cumulative masticatory activity in MVC was within the threshold and increased in BOC. The increase in masseter activity in BOC was significant $(p<0.012)$.

In Group 3, the cumulative masticatory activity in MVC and BOC was within high-normal threshold values.

\section{Discussion}

Mouthguards may have ergogenic effects [20]. The purpose of the current study was first, to determine the pinpoint accuracy (PA) with and without an MG and second, to predict possible positive or negative MG effects with an electromyographic (EMG) function test of the masticatory muscles.

The most striking result of the current study is a significant increase in PA with an MG. However, it also appears that some subjects had no or negative effects on PA with an MG. In the literature, three main theories for improved athletic performance due to improved jaw kinetics with an MG are discussed: 1 ) reduced afferent interstitial C3/4 reflexes economize cardiac workload, ventilation and metabolism; 2) co-activation of main sport-related muscles and concurrent activation potentiation; 3 ) improvement of masticatory muscle imbalances.

For theory 1, an increased activation of jaw muscles and more powerful clenching may produce a second area of force to improve whole body stability in addition to the core muscles [21]. The activation of the jaw muscles may increase the force production of sport-specific muscles and thereby improve biomechanics and motion economy [22, 23]. It has been speculated that better motion economy reduces the signal output of the muscles via reduced interstitial C3/C4 afferent activity [21,23].

For theory 2, voluntary teeth-clenching induces a co-activation of the sternocleidomastoid muscles [4, 6-8]. Clenching may also enhance muscular force due to an improved concurrent activation potentiation [20] and may increase the performance of sport- related muscles via neuromuscular responses [22].

For theory 3 , the characteristics of the occlusal surfaces, malocclusion [7, 8] and muscle imbalances [24] may impair muscle coactivation. Therefore, jaw repositioning with an MG may also enhance and improve neuromuscular responses in the sport related agonist muscles [22]. It is speculated that, through the change in dental occlusion due to an MG, the central nervous system may receive afferent impulses from the altered jaw position. These alterations of the afferent information in the periodontal ligament, temporomandibular joint or masticatory muscles may cause improvements of the upper body posture by efferent adaption or compensation patterns [1]. Julià-Sánchez et al. [15] compared the body balance on unstable platforms under opposing dental occlusion and fatigue conditions for two extreme levels of stability (stable/unstable). They concluded that the sensory information linked to dental occlusion for balance control comes strongly into effect when more difficult balance control conditions are present (i.e., unstable conditions, fatigue). This may also be relevant in volleyball where a combination of trunk stability, shoulder girdle activity, and body posture is required. The current study supports the concept of a functional coupling between the stomatognathic system and the main sport-related muscles.

Regarding possible reasons for beneficial MG effects on pinpoint accuracy (PA) in Group 1, this subgroup had a high masseter resting tone, indicating a masseter overload, i. e., a muscle weakness. This is confirmed by the low MVC activity. Overload and reduced MVC indicate an intercuspal disorder and a low jaw-clenching force. The positive MG effect can be understood by the BOC results. The cotton rolls in the $\mathrm{BOC}$ testing induce a higher intercuspal distance, which also occurs with an MG. In BOC, the masseter activity markedly increased by approximately $30 \%(p<0.04)$. The masseter profits from the pre-distension due to the mouthguard. In the context of the concurrent activation potentiation theory, this consideration would explain the positive effects in Group 1.

As to possible reasons for positive MG effects on PA in Group 2, this subgroup had a low masseter resting tone, indicating a physiological relaxation without signs of a functional disorder. The masseter activity in MVC was high-normal, indicating good muscle power. However, there was a marked difference in MVC between the right and left side, indicating a muscle imbalance. This imbalance was improved by the MG as indicated by an increase in BOC of approximately $30 \%(p<0.012)$. The jaw positioning due to the MG may also explain the improvement in PA by enhanced support of the shoulder girdle [25].

There are several possible reasons for better effects on PA in Group 1 vs. Group 2. In contrast to Group 2, Group 1 had markedly more masticatory muscle problems, such as resting overload and functional weakness, which were positively affected by the MG. So it appears that the mouthguard may have produced ergogenic effects in these groups for different reasons. Whereas in Group 1 the MG may have mainly compensated for intercuspal disorder and muscle weaknesses, in Group 2 only reflex inhibition due to muscular imbalance may have been improved. This may also explain that the MG effects were best in Group 1.

Regarding possible reasons for negative MG effects on PA in Group 3, this subgroup had no increased masseter resting tone and showed similar activities in MVC and BOC within the threshold. So these subjects had no pathologies such as overload or improper clenching and no beneficial effects would be expected from the MG. Therefore, a balanced condition of the masticatory system can be assumed. The MG presumably caused a disturbance in the neuronal muscular regulation of masticatory and peripheral muscles $[16,17]$ with impaired pinpoint accuracy.

In conclusion, the pinpoint accuracy in volleyball players was significantly better when using a mouthguard. Masticatory EMG characteristics were seen after subdividing the subjects in positive and negative mouthguard responders. It appears that an initial masticatory EMG function test may be useful to detect positive or negative mouthguard effects. When ergogenic effects are expected, the use of a mouthguard may be recommended in case of intercuspal disorders, muscle weakness or imbalance. However, this pilot trial needs more studies to clarify the hypothesis and expected results. 


\section{Acknowledgements}

We thank Susanne Schüler, Yvonne Bernwinkler, Marie Ackermann and Maik Winkler for test assistance.

\section{Conflict of Interest}

The authors declare that they have no conflict of interest.

\section{References}

[1] Ohlendorf D, Seebach K, Hoerzer S, Nigg S, Kopp S. The effects of a temporarily manipulated dental occlusion on the position of the spine: A comparison during standing and walking. Spine J 2014; 14: 2394-2391

[2] Fujimoto M, Hayakawa L, Hirano S, Watanabe I. Changes in gait stability induced by alteration of mandibular position. J Med Dent Sci 2001; 48: 131-136

[3] Miles TS, Flavel SC, Nordstrom MA. Control of human mandibular posture during locomotion. J Physiol 2004; 554: 216-226

[4] Ehrlich R, Garlick D, Ninio M. The effect of jaw clenching on the electromyographic activities of 2 neck and 2 trunk muscles. J Orofac Pain 1999; 13: 115-120

[5] Fernández-de-las-Peňas C, Carratalá-Tejada M, Luna-Oliva L, Miangolarra-Page JC. The immediate effect of hamstring muscle stretching in subjects' trigger points in the masseter muscle. J Musculoskelet Pain 2006; 14: 27-35

[6] Ormeno G, Miralles R, Santander H, Casassus R, Ferrer P, Palazzi C, Mova $\mathrm{H}$. Body position effects on sternocleidomastoid and masseter EMG pattern activity in patients undergoing occlusal splint therapy. Cranio 1997; 15: 300

[7] Santander H, Miralles R, Jimenez A, Zuniga C, Rocabado M, Moya H. Influence of stabilization occlusal splint on craniocervical relationship. Part II: Electromyographic analysis. Cranio 1994; 12: 227-233

[8] Zuniga C, Miralles R, Mena B, Montt R, Moran D, Santander H, Moya H. Influence of variation in jaw posture on sternocleidomastoid and trapezius electromyographic activity. Cranio 1995; 13: 157-162

[9] Kibana Y, Ishijima T, Hirai T. Occlusal support and head posture. J Oral Rehabil 2002; 29: 58-63

[10] Fujino S, Takahashi T, Ueno T. Influence of voluntary teeth clenching on the stabilization of postural stance disturbed by electrical stimulation of unilateral lower limb. Gait Posture 2010; 31: 122-125

[11] Hosoda M, Masuda T, Isozaki K, Takayanagi K, Sakata K, Takakuda K, Nitta O, Morita S. Effect of occlusion status on the time required for initiation of recovery in response to external disturbances in the standing position. Clin Biomech 2007; 22: 369-373
[12] Milani RS, De Perière DD, Lapeyre L, Pourreyron L. Relationship between dental occlusion and posture. Cranio 2000; 18: 127-134

[13] Bracco P, Deregibus A, Piscetta R. Effects of different jaw relations on postural stability in human subjects. Neurosci Lett 2004; 356: $228-230$

[14] Cuccia AM, Caradonna C. Binocular motility system and temporomandibular joint internal derangement: A study in adults. Am J Orthod and Dentofacial Orthop 2008; 133: 15-20

[15] Julià-Sánchez S, Alvarez-Herms ], Gatterer H, Burtscher M, Pagès T, Viscor $G$. The influence of dental occlusion on the body balance in unstable platform increases after high intensity exercise. Neurosci Lett 2016; 617: 116-121

[16] Ferrario VF, Sforza C, Serrao G, Colombo A, Schmitz JH. The effects of a single intercuspal interference on electromyographic characteristics of human masticatory muscles during maximal voluntary teeth clenching. Cranio 1999; 17: 184-188

[17] Koyano K, Ogawa T, Sumiyoshi K, Tsukiyama Y, Ichiki R, Suetsugu T. Effect of occlusal splint on masticatory movement in healthy individuals. Cranio 1997; 15: 127-131

[18] Yoshino G, Higashi K, Nakamura T. Changes in weight distribution at the feet due to occlusal supporting zone loss during clenching. Cranio 2003; $21: 271-278$

[19] Harriss DJ, Macsween A, Atkinson G. Standards for ethics in sport and exercise science research: 2018 update. Int J Sports Med 2017; 38: 1126-1131

[20] Ebben WP, Kaufmann CE, Fauth ML, Petushek E]. Kinetic analysis of concurrent activation potentiation during back squats and jump squats. J Strength Cond Res 2010; 24: 1515-1519

[21] Schulze A, Laessing J, Kwast S, Busse M. Influence of a vented mouthguard on physiological responses in handball. J Strength Cond Res 2018, May 23 doi:10.1519/JSC.0000000000002596. [Epub ahead of print]

[22] Buscà B, Moreno-Doutres D, Peňa ], Morales ], Solana-Tramunt M, Aguilera-Castells J. Effects of jaw clenching wearing customized mouthguards on agility, power and vertical jump in male high-standard basketball players. JESF 2018; 16: 5-11

[23] Schulze A, Kwast S, Busse M. Vented mouthguard effects on cardiopulmonary parameters in basketball: A pilot study. EC Dent Sci 2017: 15: 182-190

[24] Shimazaki T, Motoyoshi M, Hosoi K, Namura S. The effect of occlusal alteration and masticatory imbalance on the cervical spine. Eur ] Orthod 2003; 25: 457-463

[25] Verban EM, Groppel JL, Pfautsch EW, Ramseyer GC. The effects of a mandibular orthopedic repositioning appliance on shoulder strength. J Craniomandibular Pract 1984; 2: 232-237 